\title{
Developing a risk-adaptive technology roadmap using a Bayesian network and topic modeling under deep uncertainty
}

\author{
Yujin Jeong ${ }^{1} \cdot$ Hyejin Jang ${ }^{1} \cdot$ Byungun Yoon ${ }^{1} \mathbb{C}$
}

Received: 13 November 2019 / Accepted: 5 March 2021 / Published online: 20 March 2021

(c) Akadémiai Kiadó, Budapest, Hungary 2021

\begin{abstract}
Firms today face rapidly changing and complex environments that managers and leaders must navigate carefully because confronting these changes is directly connected with success and failure in business. In particular, business leaders are adopting a new paradigm of planning, dynamic adaptive plans, which react adaptively to uncertainties by adjusting plans according to rapid changes in circumstances. However, these dynamic plans have been applied in larger-scale industries such as wastewater management in longer-range time frames. This paper follows the dynamic adaptive plan paradigm but transfers it to the technology management context with shorter-range action plans. Based on this new paradigm of risk management and technology planning, we propose a risk-adaptive technology roadmap (TRM) that can adapt to changing complex environments. First we identify risk by topic modeling based on futuristic data and then by sentiment analysis. Second, for the derived risks, we determine new and alternative plans by co-occurrence of risk-related keywords. Third, we convert an existing TRM to network topology with adaptive plans and construct a conditional probability table for the network. Finally, we estimate posterior probability and infer it by Bayesian network by adjusting plans depending on occurrence of risk events. Based on this posterior probability, we remap the paths in the previous TRM to new maps, and we apply our proposed approach to the field of artificial intelligence to validate its feasibility. Our research contributes to the possibility of using dynamic adaptive planning with technology as well as to increase the sustainability of technology roadmapping.
\end{abstract}

Keywords Technology roadmap · Adaptation pathways $\cdot$ Bayesian network $\cdot$ Risk and uncertainty · Topic modeling

Byungun Yoon

postman3@dongguk.edu

1 Department of Industrial and Systems Engineering, College of Engineering, Dongguk University, 3-26, Pil-dong 3ga, Chung-gu, Seoul 100-715, South Korea 


\section{Introduction}

Decision makers face deep uncertainties from a wide range of viewpoints, for instance, social, technological, economic, political, and ecological. However, when faced with uncertain futures as a result of drivers such as technological, socioeconomic, and political change and corresponding policy and societal responses, the assumption that we can identify a "best-guess" output might no longer be appropriate (Haasnoot and Middelkoop, 2012; Haasnoot et al., 2013; Maier et al., 2016). This is because in such situations, there are multiple plausible future trajectories that generally correspond to distinct future states of the world that do not have an associated probability of occurrence or cannot even be ranked (Kwakkel et al., 2013). Therefore, confronting an uncertain future requires a different conceptual approach to thinking about uncertainty; this need has resulted in the development of different terms that can be used to encapsulate the concept of multiple plausible futures, of which deep uncertainty is arguably the most well-known (Lempert et al., 2004; Maier et al., 2016; Walker et al., 2013).

We used to encounter many failures in planning and forecasting because the actual future differs from the hypothesized one owing to unpredictable and uncertain events (Strauss et al., 2004). Risk and uncertainty are unexpected and require quick responses from decision makers, and, a new paradigm, dynamic adaptive plans, has recently emerged. Dynamic and adaptive plans contain a strategic vision of the future, commit actors to shortterm actions, and establish frameworks to guide future actions; these plans can cope with and react to sudden events in advance. With the emergence of this new paradigm in planning and forecasting, researchers devoted much attention to scenario-based technology roadmapping and adaptive policymaking because the new plans allow for considering a wide range of risks under deep uncertainty.

Technology is continuously evolving, driven by R\&D activities and by the demands of consumers or corporates for new products or applications. Firms seek to understand the nature of technology evolution in order to create accurate forecasts, take advantage of investment and market opportunities, and maintain or grow market shares. An ecosystem view is useful to represent many technologies and their relationships that make up technology landscape. In the ecosystem that was usually utilized in biological science, specific species evolve with affecting to other species and influenced by others and external forces. Since the first introduction of ecosystem view, there have been several metaphors such as science, technology, innovation and business ecosystems. By considering the technology ecosystem as an inter-related set of technologies, a manager is able to identify factors that have an impact on innovation, development and adoption of new technologies and ultimately the success of the business activities that use the innovations (Adomavicius et al., 2008). Innovation ecosystem and business ecosystem has more extensive range of concepts including interrelationships with organization and society than technology systems. Likewise, these ecosystem views are similar to the components of TRMs-technology, product, and market. TRMs can show the overall landscape of each components and their evolution pathways for satisfying firm's objectives and visions.

Scenario-based (or scenario-driven) TRMs have attracted a great deal of recent attention for their responsiveness to deep uncertainty. The maps have conventionally been challenging with regard to rapidly responding to changes owing to their straight-line (i.e., single) scenarios. Scenario planning overcomes this limitation of traditional technology roadmapping by capturing the full contexts of decisions, enabling anticipation of a broad range of possible changes. Another concept for handling deep uncertainty is adaptive policymaking, 
which suggests that policies be treated as experiments with the aim of promoting continual learning and adaptation in response to experience. Adaptive policymaking developed through workshops and exploratory modeling and analysis with the goal of responding to deep uncertainty at a balance between over- and underreacting to circumstances (Buurman \& Babovic, 2016; Kwakke et al., 2013) with an adaptive robust design approach (Hamarat et al., 2013).

Although researchers attempted to establish planning and forecasting under deep uncertainty, there were limitations. In terms of scenario-based TRMs, most investigators focused on scenario discovery and development by identifying risk factors through environmental scanning; even when scenario discovery and development preceded planning and forecasting, there was a lack of connection between scenario planning and TRMs (that is, the results of technology or market planning). Moreover, traditional TRMs could not reevaluate and update in response to unexpected and complicated changes in external and internal environments; it was difficult to update established maps because they were so time consuming to assemble initially. In fact, traditional TRMs have mainly been developed in workshops with domain experts, but such meetings require considerable resources, making them difficult to assemble quickly for rapid responses to events. Adaptive policymaking has been limited in application to areas such as water and transportation, considered large industries.

Therefore, with this paper we aimed to suggest a new concept called the risk-adaptive technology roadmap through a Bayesian network and topic modeling. This map enables rapid responses to unexpected events by adapting strategies or paths composed of technology, product, and market. The proposed roadmap seeks to monitor occurrence of risky events in real time to trigger adaptive responses. To develop the risk-adaptive TRM, we first detected risk events with an existing map as well as trends in key word occurrence. For this paper, we define risks as all events that have positive and negative effect on the future. Second, we estimated potential consequences as an impact and likelihood through topic modeling and keyword analysis. The impact will be treated as targets for the suggested roadmap after forecasting risk events. Third, we explored possible and necessary actions to achieve these targets by co-occurrence of words from the viewpoints of technology, product, and market with the aim of deriving the elements—nodes, links, and time-of the technology roadmap. Then, we estimated the probability and impact of each action for efficiently arriving at the targets by Bayesian network. Finally, we mapped the results in each phase on the new roadmap, suggesting the main path as well as alternative paths based on total utility and the extent of risk. In particular, this roadmap can provide the point where a user needs to make a decision to achieve desired objectives.

This study highlights adaptive planning to rapidly changing environments that affect the innovation ecosystem. For this reason, we choose three main methodologies, topic modeling, sentiment analysis and Bayesian network. It is inevitable because technology roadmapping requires several tasks for achieving individual goals interacting with each otheridentifying possible risk events, evaluating the degree of risk, and analyzing relations between the risks and each element in TRM (technology, product, and market). The topic modeling has been widely used to analyze a large number of documents and the Bayesian network has shown the high performance to identify relationships between events (or objects). Although three types of methodology have been applied in the past, most of the previous studies more focused on analyzing technology itself or tracking research (Lee \& Song, 2020; Li \& Porter, 2018; Marrone, 2020; Ranaei et al., 2020) rather than the context of future planning such as technology roadmap. Besides, Even though they have been respectively utilized on the basis of their performance within different areas, a few studies 
are worked with Bayesian network and topic modeling. In contrast with previous studies, the topic modeling and the Bayesian network are appropriate to derive future events from large databases and to evaluate interrelation between future risk events and TRM that was established in the past. In particular, topic modeling in this study contributes to perceive and prepare the sudden risks in advance as much as possible by analyzing the futuristic databases where the scenarios for the distant future are described. Bayesian network also assists to estimate impacts caused by the risks derived from futuristic databases using topic modeling, which results in changing overall strategies through adjusting the contents of the nodes and relations between nodes in the technology roadmap. Consequently, our paper can be utilized to sustain the established technology roadmap where lots of resources have been already used for roadmapping through updating several elements in the established roadmap. Moreover, by analyzing different databases for generating each layer of TRM, we also emphasized close relatedness between each layer of TRM and the innovation ecosystem. The technology ecosystem and innovation ecosystem are directly connected and represented to the technology, product, and market layers on TRM.

The remainder of this study is divided into four sections. "Background" section provides the review of the historical research on TRMs and descriptions of our methodologies for estimating probability and impact of risk as well as of Bayesian networks and influence diagrams. In "Research process" section we show the basic concept and overall process for developing the risk-adaptive technology roadmap and then apply it to self-driving automobiles to illustrate the suggested approach in "Results" section. In "Conclusion" section, we provide final academic and managerial insights based on our results.

\section{Background}

\section{Technology roadmaps}

TRMs are powerful planning tools for exploring and communicating dynamic linkages between technology resources, organizational objectives, and changing environments (Phaal, 2004). Researchers have defined the technology roadmap as a consensus on visions of future technology landscapes that offer ways to identify, evaluate, and select strategic alternatives for achieving specific objectives (Kostoff \& Schaller, 2001; Phaal et al., 2004, 2005). Rinne (2004) described these maps as evolution maps of technologies and the products that incorporate them, and Garcia and Bray (1997) considered them documents that identify critical system requirements, product and process performance targets, and technology alternatives and milestones for meeting those targets. TRMs enable to capture a high level, synthesized and integrated view of strategic plans in a simple and graphical format. They seek to answer three simple questions by considering a range of different perspectives including market, products, and technology-(1) where are we going? (2) where are we now? (3) how can we get there? (Phaal et al., 2007). TRMs can be applied at various levels of granularity, focusing on from components of complex systems, to entire sectors or fields or science. The architecture of TRM is configured to suit the focus and scope of issues being addressed, to provide a framework to support the dialogue necessary to develop and implement the desired innovation, strategy or policy (Phaal \& Muller, 2009). The structure of TRM is usually composed of three layers, technology, product, and market (typically the vertical axis) with time frame (typically the horizontal axis). According to firm's objective and vision, future needs are mapped and visualized on the TRM in the 
form of pathways by connecting each node (or box) based on causal relationships as shown in Fig. 1. TRMs are able to support the development and implementation of integrated strategic business, product and technology plans, providing companies with the information, process and tools to produce them.

According to increases in complexity and diversity of technology, technology and innovation is not achieved in isolation, so it co-evolves with the interaction between different entities or actors in system. From this perspective, the concept of ecosystem was emerged and has been dealt with in wide range of studies such as science ecosystem, technology ecosystem, innovation and business ecosystem in the literature on strategy and innovation. The innovation ecosystem has been defined as the complex relationships that are formed between actors or entities whose functional goal makes technology innovation possible (Jackson, 2011). The actors contain the material resources and the human capital that make up the institutional entities participating in the ecosystem. Carayannis and Campbell (2009) defined innovation ecosystem as that people, culture and technology meet and interact to catalyze creativity, trigger invention and accelerate innovation. Li (2009) utilized the business ecosystem that is an increasing focus of a firm's business strategy and can provide an emerging orientation to create novelty in business operations. Among these diverse concepts, there are common features: (1) multiple relations with other actors and entities, and (2) capability for value capture and creation. These features are strongly related to the concept of TRMs which are basically composed of three layers - technology, product/service, and business/market, and they are closely interrelated and interacts with each other. TRMs allow the evolution within each layer to be explored, together with the interlayer dependencies, facilitating the integration of technology into products, services, and business systems (Phaal et al., 2004). The ecosystem of all relevant technologies can be complex, with technologies playing multiple roles and having multiple relationships (Adomavicius et al., 2007). In particular, emerging technologies usually start from the scientific research phase, and then move to technology development phase before knowledge can be converted into commercial value through business activities. The interplays between science, technology,

\section{Unit of analysis}

\section{Roadmap}

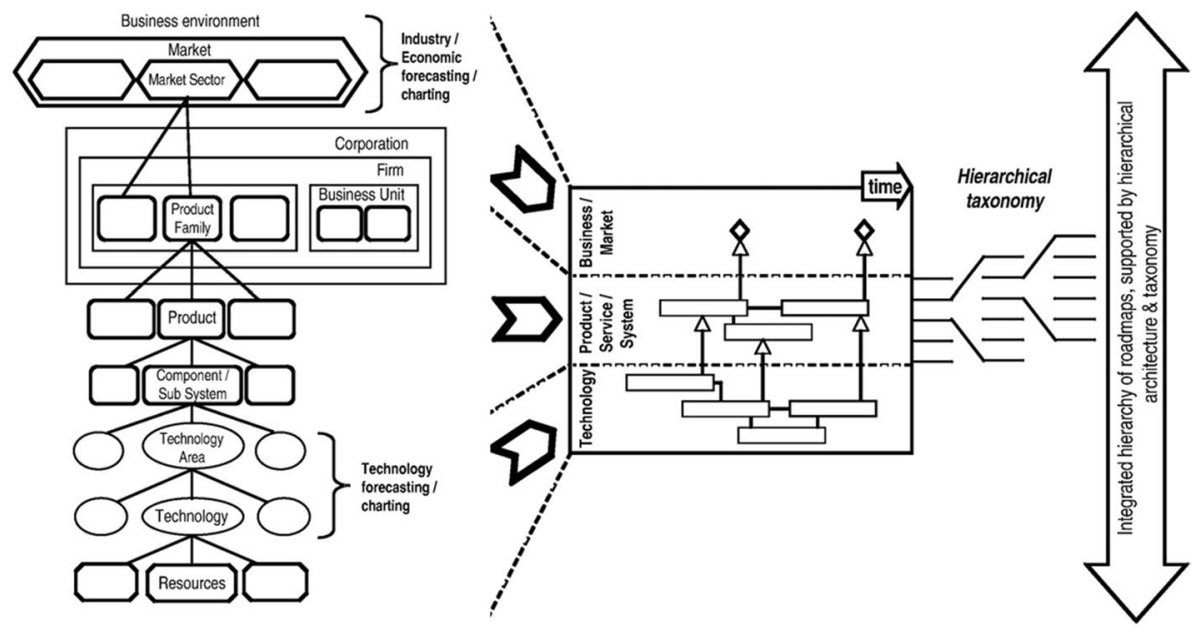

Fig. 1 Hierarchical taxonomies underpin the roadmap architecture (Phaal \& Muller, 2009) 
and business are dynamic and requires better understanding of ecosystem. These ecosystems correspond to each layer in TRMs. For example, science and technology ecosystems focus on basic technology and applied technology respectively (Xu et al., 2018) and these technologies can be mapped and explored in the technology layer in TRMs. The product or service using them is mapped on the product layer of TRMs. The drivers or trends in the market are mapped on business/market layer, which is closely related to innovation and business ecosystem. The elements comprising TRMs seems to be limited to their contents, but environmental factors and other network, resources also can be dealt with in TRM layers. In other words, TRMs are appropriate to explore and represent science, technology, and business ecosystems by analyzing knowledge, technology, and innovation and exploring their pathways.

Since Motorola introduced the concept of TRMs in the 1980s, they have received much research attention in the field of technology planning and forecasting. Initially, these plans were established in intensive workshops with groups of domain experts in wide ranges of industries, but one outcome of this process was that technology roadmapping required significant time and effort for new workshops whenever there was new technology or a new market (Park et al., 2020). Even when expert-based roadmapping led to more accurate and practical results, the process was costly and sometimes difficult. To overcome these limitations, researchers have made numerous, extensive attempts at exquisite roadmapping using bibliographic data such as patents and techniques such as text mining (Lahoti et al., 2018; Zhang et al., 2013), fuzzy cognitive mapping (Amer et al., 2016; Kim et al., 2016), quality function deployment (Jin et al., 2015), association rule mining (ARM) (Geum et al., 2015) and Bayesian networks (Lee et al., 2015). Technology roadmapping based on quantitative analysis using bibliographic data helps to support reliable decision-making and improves efficiency by minimizing the impacts of subjective opinions. Lee and Park (2005) suggested modularizing roadmaps for mass customization, facilitating different maps for different purposes such as forecasting, planning, and administration.

However, TRMs based on quantitative analysis greatly depend on data, making it possible to underestimate complex and uncertain environments. For this reason, multiple researchers have investigated validating the credibility of roadmapping based on quantitative analysis. To reflect the uncertainty and complexity of environments, researchers now make roadmaps more concrete with consideration of environmental factors by applying scenario analysis (Amer et al., 2016; Firat et al., 2008; Geum et al., 2014; Hansen et al., 2016; Lee et al., 2017; Siebelink et al., 2016). We summarized a history of TRM studies by reviewing previous papers and classified three generations of TRMs as shown in Fig. 2 with the intention of developing a new technology roadmap to overcome the abovementioned limitations of previous TRMs by considering complex and uncertain environments in forecasting and planning. The proposed risk-adaptive technology roadmap can be regarded as a framework for dynamic adaptive planning by detecting future risks and reacting them adaptively. The risk-adaptive concept is to monitor and extract risk events from futuristic databases and then to provide plans for addressing the events (Table 1). The risk-adaptive technology roadmap identifies potential risk events from futuristic databases describing status for the distant future and estimates their impact in the future. Then, by combining the risks and their impact, technology roadmap and trajectories linking each node in the roadmap are updated and remodeled in accordance with the possible risks. In contrast to conventional technology roadmaps that are developed at the specific time point and are not renewed in the light of changing circumstances, the proposed approach for riskadaptive roadmapping can help to revise and update the established roadmap while considering future events that may have an impact on innovation ecosystems. 


\begin{tabular}{|c|c|c|c|}
\hline $\begin{array}{l}\text { Generation } \\
\text { ofTRM }\end{array}$ & $1^{\text {st }}$ generation & $2^{\text {nd }}$ generation & $3^{\text {dd }}$ generation \\
\hline \multirow[t]{2}{*}{$\begin{array}{l}\text { Summary of } \\
\text { researdhtrend }\end{array}$} & $\begin{array}{l}\text { - Overall structure of TRM } \\
\text { TRM established by } \\
\text { workshop with group of } \\
\text { domainexperts } \\
\text { - Suggesting T-Plan for } \\
\text { increasing applicability in } \\
\text { practices }\end{array}$ & $\begin{array}{l}\text { - Elaborating process for } \\
\text { roadmapping } \\
\text { Roadmapping by } \\
\text { analyzing bibliometric } \\
\text { information } \\
\text { quantitatively and } \\
\text { systematically }\end{array}$ & $\begin{array}{l}\text { Validating credibility of } \\
\text { roadmapping based on } \\
\text { quantitative analysis } \\
\text { Concretizing } \\
\text { environmental factors } \\
\text { and applying to scenario } \\
\text { planning }\end{array}$ \\
\hline & $\downarrow$ & $\downarrow$ & $\downarrow$ \\
\hline Limitations & $\begin{array}{l}\text { Much time and effort } \\
\text { consuming because of } \\
\text { depending on opinions } \\
\text { by experts and extensive } \\
\text { workshops }\end{array}$ & $\begin{array}{l}\text { - Arguments on credibility } \\
\text { of roadmapping results } \\
\text { becauseithighly depends } \\
\text { ondata } \\
\text { - Underestimate complex } \\
\text { environment }\end{array}$ & $\begin{array}{l}\text { - Nofeedbacks by dhanges } \\
\text { onenvironments } \\
\text { - Difficulttomaintenance } \\
\text { - Not reflecting risk } \\
\text { dynamics }\end{array}$ \\
\hline
\end{tabular}

Fig. 2 The history of roadmapping studies

\section{Risk management}

During the last three decades, risk management has emerged as an essential and relevant tool in our daily lives. Aven (2012) defines risk in terms of expected value (loss), probability of an undesirable event, objective uncertainty, uncertainty, potential or possibility of a loss, probability of scenario, severity of consequences, and consequences (damage or severity) of these including uncertainty. Initially, Aven defined risk as fundamentally based on probability - that is, uncertainty expressed under the rules of probability calculus but then expanded the focus of the concept to include expected value, consequence, and the effect of uncertainty on objectives (Aven, 2012). Of these, we concentrated with this study on probability, consequences, and severity of consequences, which are common features of risk as a concept. Kaplan and Garrick (1981) defined risk analysis as composed of answering the following three questions: (1) What can happen?, (2) how likely is it that will happen?, and (3) if it does happen, what are the consequences? This can be expressed as a triplet $\left(s_{i}, p_{i}, x_{i}\right)$ where $s_{i}$ is an identified scenario, $p_{i}$ is the probability of that scenario, and $x_{i}$ is the consequence or evaluation measure of that scenario. The authors then defined risk as the set of these triplets (Kaplan \& Garrick, 1981).

Similar to this definition, we define scenarios as future events that derive from analyzing futuristic databases. These future events affect the processes of developing and planning new technologies, and we can quantitatively estimate the impacts of these events. We also predict the consequences of future events and identify the adaptive plans for responding to them, which we describe in detail in "Research process" section.

With increases in risk and uncertainty, a new planning paradigm called dynamic adaptive planning has emerged for responding to these deep uncertainties. It is necessary for when environmental conditions as well as societal perspectives and preferences change over time, including stakeholders' interest and their evaluation of plans (Haasnoot et al., 2013; Offermans, 2012; van der Brugge et al., 2005). Adaptation over the course of time is determined not only by what is known or anticipated at present but also by what is experienced and learned as the future unfolds (Yohe, 2000). Dynamic adaptive plans include strategic visions for the future, short-term actions, and frameworks to guide future actions (Albrechts, 2004; Ranger et al., 2010). 


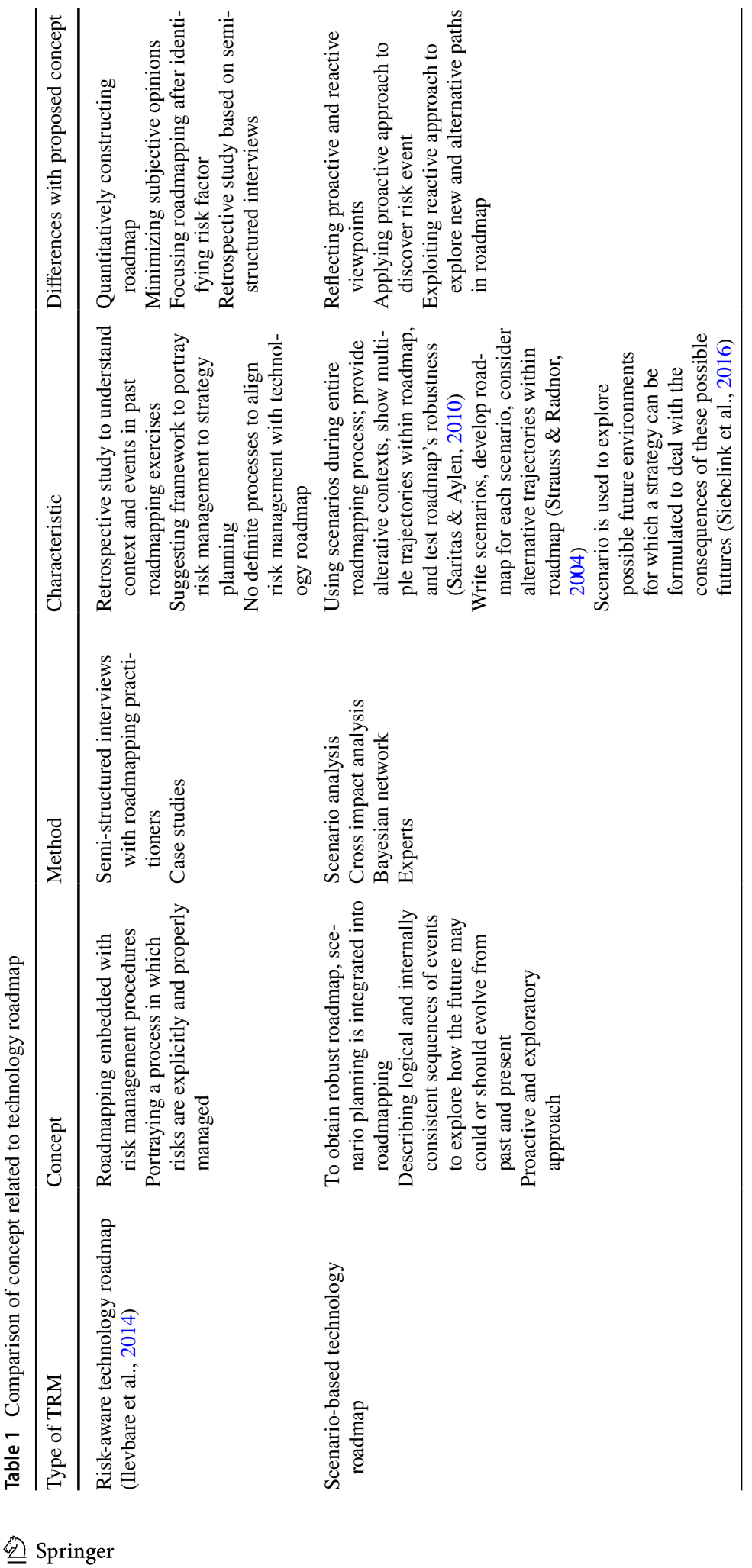




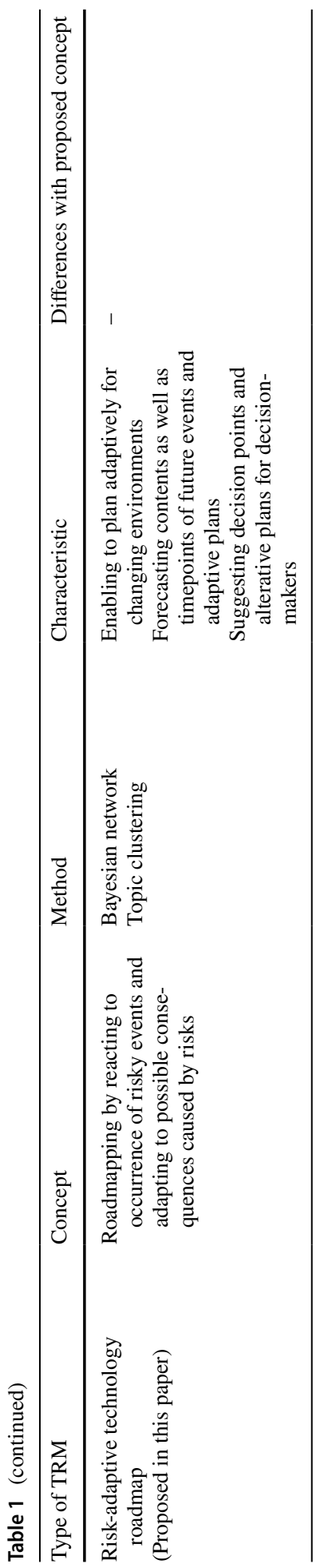


Numerous researchers have studied strategies for adapting to environmental changes (Butler et al., 2015; Buurman \& Babovic, 2016; Rosenzweig et al., 2014) and energy transitions (Hamarat et al., 2013). In particular, dynamic adaptive policy pathways, which are based on two approaches for designing adaptive plans — adaptive policymaking and adaptation pathways (DAPPs) - include transient scenarios that represent relevant uncertainties and their development over time; actions to handle vulnerabilities and opportunities; adaptation pathways describing sequences of promising actions; and a monitoring system with related contingency actions to keep the plan on the track of preferred pathway (Haasnoot et al., 2013). Within the DAPP approach, a plan is conceptualized as a series of actions over time and the essence is the proactive planning for flexible adaptations over time, in response to how the future actually unfolds. It is similar to the concept of TRMs, which present paths for exploring new technology or markets that satisfy future needs, in that DAPPs are suitable for planning long-term strategies in large-scale areas such as climate change, water management, and flood risk management (Kabir et al., 2017; Lawrence \& Haasnoot, 2017; Maier et al., 2016). Technology life cycles are shortening with time, and now, medium-term strategies are required for coping with rapidly changing environmental factors. While TRMs are appropriate for medium-term planning, they are not flexible as the landscape changes because changes to an established map require additional gatherings of experts participate at considerable efforts and costs. Within this framework, we suggest a risk-adaptive TRM that adapts strategies or paths by connecting technology, product and market when risk events occur. After identifying possible risk events from futuristic databases, we explore necessary actions to achieve goals by responding to risk events in the future through (1) monitoring occurrence of risk events, (2) analyzing correlations between risk events and elements of TRMs, (3) adapting strategies and changing paths, and (4) establishing the new TRM concept.

\section{Bayesian network}

For risk management, there were many approaches and methodologies to assess and respond to risks by qualitative as well as quantitative techniques such as system dynamics (Wang et al, 2017), scenario trees (Gailis et al., 2014), fault tree analysis (Islam et al., 2017; Purba et al., 2015), and Bayesian networks (Abaei, 2017; Kruger \& Lakes, 2015; Khakzad et al., 2013; Khakzad, 2015; Yet et al., 2016). Among these, Bayesian networks are powerful tools for making probabilistic inferences in complex domains with large numbers of variables (Constantinou et al., 2012); as such, they are widely used in risk and safety analysis based on probabilistic and uncertain knowledge. Bayesian networks are graphic probabilistic models that are composed of a graphical structure and parameters of conditional probability distributions corresponding to the structure. The graphical structure of a Bayesian network consists of nodes representing variables and arcs representing the relationships between variables; the parameters of a Bayesian network show the nature and strength of the relationships represented by the arcs. They take advantage of Bayes's theorem to update the prior occurrence probability of events given new information, called evidence $\mathrm{E}$, thus yielding posteriors. This new information usually becomes available during the operational life of a process, including occurrence or non-occurrence of accident or primary events:

$$
\mathrm{P}(U \mid E)=\frac{P(U, E)}{P(E)}=\frac{P(U, E)}{\sum_{U} P(U, E)}
$$

We used a Bayesian network to calculate costs and benefits based on multiple causal factors including the effect of individual risk factors, budget deficits, and time value 
discounting, considering the parameter uncertainty of all continuous variables (Yet et al., 2016). Ashrafi et al. (2017) implemented critical paths and extended reasoning to include the causes of delays, and de Melo and Sanchez (2008) assessed risk and predicted delays in software maintenance projects. Hu et al. (2013) utilized constraint-based structure learning algorithms on Bayesian networks to learn causal relations and make predictions about risk factors of software development projects.

For our study we exploited a Bayesian network to estimate probability of entering future markets in that this methodology is well-suited to model the attributes of uncertainty and risk that are common to projects (Yet et al., 2016). It is striking in reasoning about uncertainty as they can represent and make inference about complex joint probability distributions with numerous random variables. The suggested roadmap consists of many variables in complex environments for technology development and the structure is similar to networks. Thus, a Bayesian network is appropriate for analyzing risks in technology roadmapping under deep uncertainty.

\section{Research process}

\section{Basic concept}

Our study consists of four steps to establish the new concept of a risk-adaptive TRM within the framework of risk management: (1) risk identification, (2) risk assessment, (3) risk treatment, and (4) re-roadmapping (Fig. 3). First, we identified risks with high possibility of future occurrence by topic modeling based on futuristic databases and reevaluated identified topics by definitions of risk such as growth rate and probability. Second, we investigated impacts of risk and the relationships between TRM elements by Bayesian network. At this time, we examined not only the relationships between elements but also between

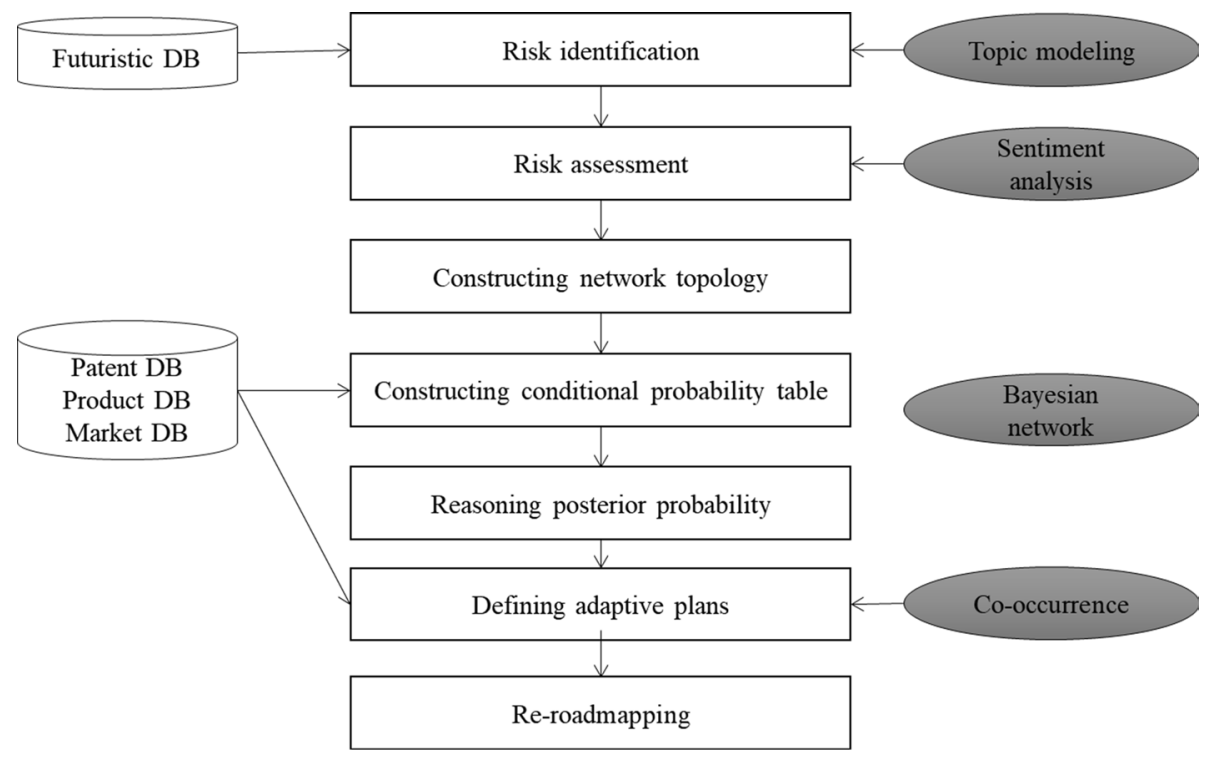

Fig. 3 Research framework 
elements and risks for identify which technology, product, or market can be influenced when future risks occur. The Bayesian network is able to show relationships between all elements including risk and to estimate final impact from risk to market based on preexamined relationships. We estimated the degree of impact by risk, and we updated posterior probability considering deep uncertainty. Third, we determined the alternative paths to cope with risk with the aim of maximizing utility and market access by comparing metrics for structural and data feasibility. Finally, we mapped these as nodes and arcs on a TRM similar to a Bayesian network. Nodes and arcs on a Bayesian network are paths connecting technology, product, and market nodes in the risk-adaptive TRM, and the alternative paths in the prior step can represent substitute TRM trajectories. The new map supports technology planning by adapting to future risk factors and establishing alternative strategies based on objective evidences. The nodes on each layer in TRM are derived by analyzing different databases such as patents, research papers, market report, futuristic databases for explaining and mapping science, technology and business ecosystem on TRM. The technology ecosystem, composed of a set of emerging technologies, is mapped on technology layers of the TRM by analyzing patents and research papers using topic modeling (LDA). The product and services using applied technologies that is a component of business ecosystem are mapped on the layer of products in the TRM. Future markets are finally represented on the market layer by analyzing market reports that considers market trends and actors related to the business ecosystem. We explain our detailed process for developing the risk-adaptive TRM below.

\section{Overall process}

\section{Risk identification}

As we mentioned earlier, a risk can be defined as an uncertain event or circumstance that influences the future (ISO's definition) and consists of three parts: scenario (risk events in this paper), possibility, and consequence. First, risk events (or scenarios) can be derived from future databases, which gather experts' visions related to future markets or promising high technology from diverse perspectives such as sustainable energy, artificial machines, and rewriting life. In this point of view, 'the risk-related words' are defined as the words that describe risk events, and they were derived by analyzing the futuristic databases. In this regard, we assumed the futuristic database contains information related to events or issues in the future, so this assumption leads to the words that are related to the risk events. Analyzing futuristic databases helps to find future events or possible scenarios (previous research) as well as provide insights for the future, so we utilized them for this study to identify possible risks in the near future such as Nextbigfuture (https://www.nextbigfuture. com/), MIT Technology Review (https://www.technologyreview.com), and FutureTimeline (https://www.futuretimeline.net). The futuristic databases contain blueprints and scenarios for the future by experts for future foresight and forecasting. They show how worldwide population is changed and which technology will be emerged in the future within various fields (e.g. artificial intelligence, quantum computers) or how the life-style of human being will be changed in the future and so on. For example, in the Future Timeline, a website for forecasting the future, they suggest the future status by each year from different viewpoints such that "Global population is reaching a crisis point in 2030", "The 6G standard is released in 2030", "Terabit internet speeds are a common place in 2032", "Swarm robotics are reaching the nanometer scale in 2035-2040", "Nanofabricators are a mainstream 
consumer product in 2062". After we collected the futuristic data, we used latent Dirichlet allocation (LDA), the most popular method for clustering data from many documents, to extract future risks. LDA derives topics and key words from document and word distributions by considering relationships between words, meaning contexts. Other elements for defining risk are possibility and consequence, and we estimated these with the LDA.

The possibility of risk refers to the likelihood that risks will occur in the future, and it can be estimated based on the frequency of keywords related to risk events; if risk-relevant words appear frequently, an event related these words will occur in the near future. To quantitatively estimate consequences of risk, we applied sentiment analysis, which entails evaluating sentiments as positive or negative. Sentiments can be interpreted as impacts of risk events: for example, negative sentiments about a topic indicate that an event may cause negative consequences. This paper classified risk events as just two classifiers, positive and negative, and used the results focusing on the absolute degree of impact because this phase aimed to identify the most influential risk events to the future. Especially the most influential risk event enables to make huge profit by responding at the right timing or earlier timing than other firms, or causes economic loss by missing opportunities. Thus, we defined future risk events with low probability but high impact as definitive future risks. The most influential risk which has high absolute value of impact has high priority and it requires more strict management and adaptive planning in the future.

\section{Risk assessment}

We assessed risk by keyword analysis and Bayesian network by analyzing the relationships between elements of the TRM and risks; this is a critical step for estimating risk impacts and deriving alterative paths to cope with future risk. To do this, we first investigated the relationships between TRM nodes and risk by similarity between key words of each representative document such as patent, product manual, market report, and future data. Each node had strong relationships with distinct documents, for example, information related to the technology node included patent documents, and the product nodes were relevant to product documents. Thus, we estimated risk impacts by growth rate, co-occurrence, and cosine similarity between keywords related to risk events and documents related to each node on TRMs. As shown in Table 2, the tertile value is used to conclude the criteria to discriminate the state of each node-low, medium, and high—on Bayesian network, and the mean values of each state of risk, technology, product, and market nodes on TRM are presented in Table 2 .

\section{Definition of risk-adaptive plans}

Based on risk from futuristic database, we extracted the consequences by analyzing associations between risk and nodes in the technology roadmap. We aimed to find which risks would affected the TRM elements-technology, product, and market-because understanding the relationships between them are fundamental for exploring adaptive plans for risk; in the previous step we quantitatively estimated the impacts, so in this step we analyzed the consequences, and this association analysis leads to links between nodes and risk events. We measured two different types of association, between nodes and risk and between risks, based on similarities between relevant documents and a linking grid; if the words related to nodes and risks frequently occurred together, they had similar features and high association between them, and we further interpreted that 


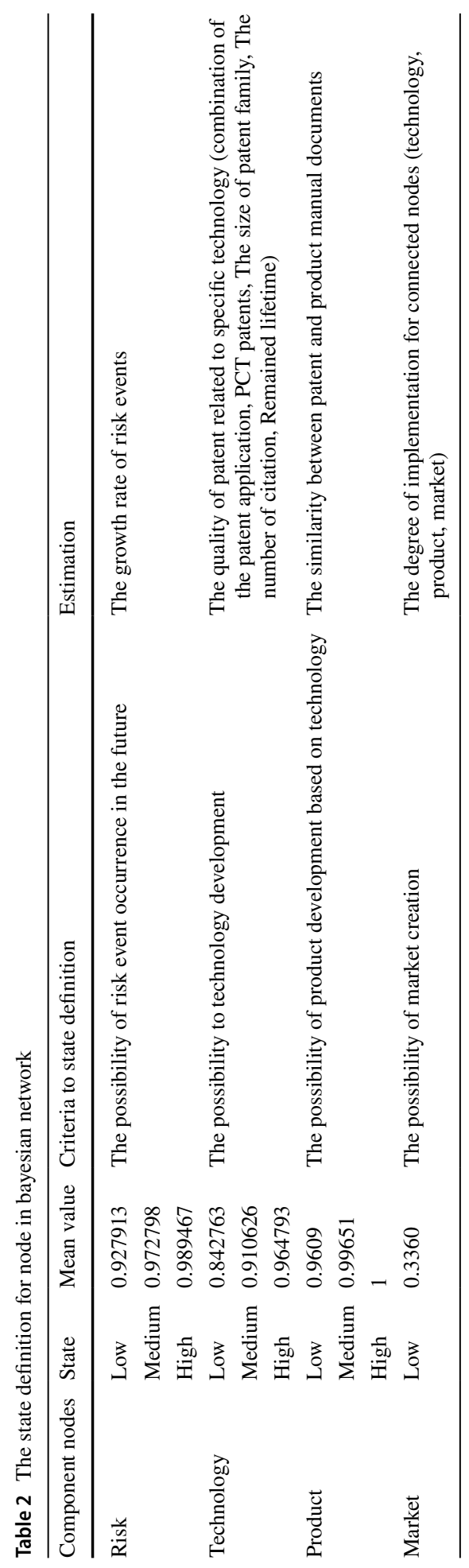


they had influential relationships with each other. The features of each node are well represented in documents, and representative or relevant opinions about risks are shown in the futuristic databases.

For each element that had an association with risk, we explored their plans for next actions to adapt and respond to risk occurrence using two approaches, generating new ideas using SCAMPER and cosine similarity between risk and TRM elements to adjust the present paths in the technology roadmap. When a risk event happens and affects technology, a product, or a market, the next action plans are discovered in advance; the adaptive plan offers alternative plans and solutions for addressing risk events that bring about changes in corporate strategy. First, SCAMPER is an approach to new idea generation that supports exploring new plans of existing nodes related to risks. After the possibility of risk events is identified, their contents are derived by SCAMPER with text mining, and this process results in new TRM nodes and links. Second, the path is changed without creating novel nodes within the framework of risk management that aims to find optimal solutions with minimizing loss as well as maximizing utility under given conditions. For that, the roadmap paths can be adjusted for achieving optimal solutions through a Bayesian network.

Bayesian networks enable posterior probability by Bayes rule based on conditional probability tables for each node and chain rule. The conditional probability table is usually defined or learned by by experts' scores or data that can explain the possibility of nodes in the Bayesian network. For this paper, we utilized the documents that were relevant to each node in the roadmap; for instance, technological specifications and features are mainly described in patent documents, and product and market data are also depicted in reports. We estimated all elements quantitatively as shown in Table 2, and they construct the conditional probability table. We finally estimated the posterior probability by chain rule; in this paper, this probability refers to market attainability, which is the end node of the paths in the technology roadmap. Finally, the path from technology to market including risks are established by simulations in accordance with whether the risk occurred or not.

\section{Technology roadmapping}

Based on the relationships between nodes and risk with adaptive plans, the existing technology roadmap is rearranged and updated by risks; the risk is mapped on the upper side of the map, and new nodes derived as adaptive plans are also mapped. The basic structure of TRM is composed of three layers - technology, product, and market layers. Then, the node that was mapped on the existing roadmap but needs to change direction toward the next node based on the adaptive plan. New adaptive plans are represented as dotted nodes, and the subsequent results from risk and adaptive plans are connected dotted lines. Multiple nodes that have multiple adaptive plans are depicted as decision nodes, which supports users in selecting strategies. Each layer composed of multiple nodes correspond to technology ecosystem and innovation ecosystem. In the technology layer, emerging technologies are mapped and the set of technology shows science and technology ecosystem focused on basic technology. In the product layer, future products and services are mapped by relying on applied technology, which is reflecting the innovation ecosystem. Future market drivers and trends are mapped on the market layer in the TRM. This layer presents and maps the business ecosystem with future risk events extracted at the initial phase of this study. For this paper, technology roadmapping included not only creating new nodes and paths but also updating the previous technology roadmap to increase sustainability. 


\section{Results}

\section{Data collection}

In order to illustrate our proposed approach, we selected an advanced driver assistance system, which is a core technology for self-driving cars, for this paper. In the context of Industry 4.0, which is founded on artificial intelligence, autonomous cars have received attention as next-generation vehicles that combine information and communication technology. Most traffic accidents are caused by driver errors, so the aim with self-driving cars is to prevent mistakes and reduce traffic accidents. However, this recent technology has very short life cycles and is surrounded by a number of environmental factors and risks; this context makes adaptive and dynamic planning through risk-adaptive roadmapping that can address risk events as they occur in real time.

\section{Risk identification}

To identify risk events, we collected 13,032 units of future data from three futuristic databases-Kurzweil, MIT Technology Review, and NextBigFuture, by web crawling. Two of the databases divided some data by specific classification, and Kurzweil has no classification for future data. We used data from the last three years to identify future possible risk, and after we collected the data, we preprocessed all documents such as by removing unnecessary words. We then clustered these cleaned data and used LDA to extract topics for each cluster. We used the ldatuning package in $\mathrm{R}$ to define the number of topics, ultimately arriving at 180 topics from the futuristic databases. As we discussed earlier, each topic can be defined as an event (or scenario) with a high risk of occurring in the future and that affects a technology development path. Among these, we analyzed for this study the topics that were of high priority for developing new technologies. LDA provided the topics and keywords in each topic cluster, which became the baseline data for estimating risk components such as probability (recent increase in word frequency) and future impact (sentimental value of each topic). We calculated sentimental value using SentiWordNet and identified the following topics as having high possibility and high impact, labeling them high-priority risks: climate change, cloud computing, space and aircraft, cryptocurrency, Internet of Things, voice recognition, and energy (in Table 3).

\section{Risk analysis}

To assess the risk using the Bayesian network, we first converted each node in the existing TRM to a node on the network and connected them with each other by predefined arcs in the technology roadmap. We represented the risk derived from the prior as network topology connected with TRM nodes based on correlations between them. Then, we calculated the conditional probability based on the nodes' states driven by data related to technology, product, and market. For the risk nodes, we calculated the degree of risk as the yearly average increase in futuristic data generated recently, which was the main source of data for this study. For the technology nodes, five indicators were defined from patents: patent application, patent cooperation treaty, number of family patents, number of citations, and remaining duration of patents; combining these indicators, we assessed each technology as high, medium, or low. For the product nodes, we collected documents such as user guides, manuals, and technical reports related to each product or system, and this was similar for 


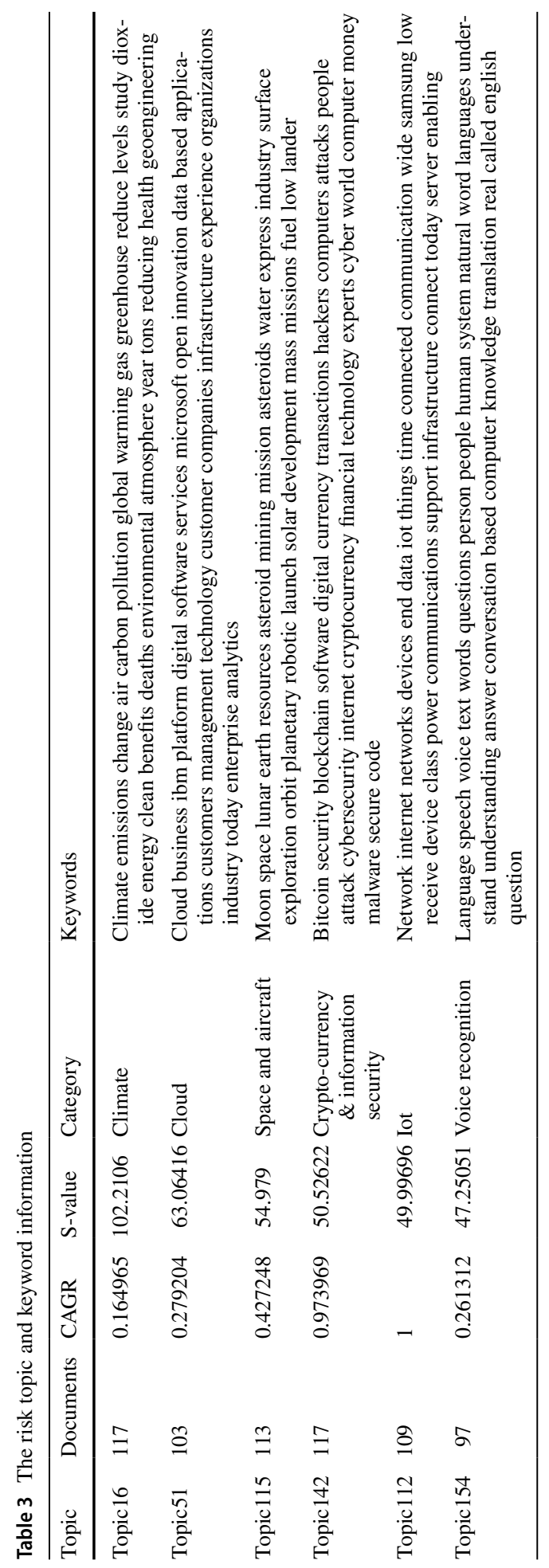


the market nodes, especially market forecast reports for autonomous vehicles. We vectorized all technology, product, and market documents and then estimated by cosine similarity between documents; the similarity between documents estimates the occurrence probability of a given specific situation, and thus, we selected this value for this study as proxy measure with minimal researcher intervention. We finally determined conditional probability based on the degree of each node. Specifically, we used Genie software and calculated the marginal posterior probabilities and results in the re-roadmapping; after we estimated the probabilities for the existing nodes and relationships in the established roadmap, we added the risk events and investigated the changes or adaptive plans.

Of the multiple risk events we identified, we mapped four onto the TRM risk layer: climate change, cloud computing, voice recognition, and Internet of Things. These risks affect all nodes on all layers in the roadmap, so that they are connected with each node; by assuming events related to these four risks will happen in the future, and future plans can be adapted to changes caused by the risks of the events. As shown in Fig. 4, if the risk event has not occurred yet, the probability of entering the future driverless car market is not high; the possibilities of entering markets in the future were medium or low, which requires greater efforts for technology or product development. Based on the risk events extracted from the prior step, there was no change at M1 (Level 3 self-driving car market), but the possibility at M2 (Level 4 self-driving car market) was slightly low. With the aim of penetrating future markets, we assumed that all risk events would occur at once in the near future and that the possibility of entering M1 and M2 is 100\%. Then, we observed changes in probability for each node in the product and technology layers as shown in Table 4 and Fig. 5, and we identified higher probabilities for development of T2 (pedestrian and object detection), T5 (cooperative adaptive cruise control), and T6 (lane keeping assistance system) by occurrence of risks. On the contrary, for T1 (autonomous emergency braking), T3 (pedestrian collision warning system), and T4 (lane detection), and T7(Emergency

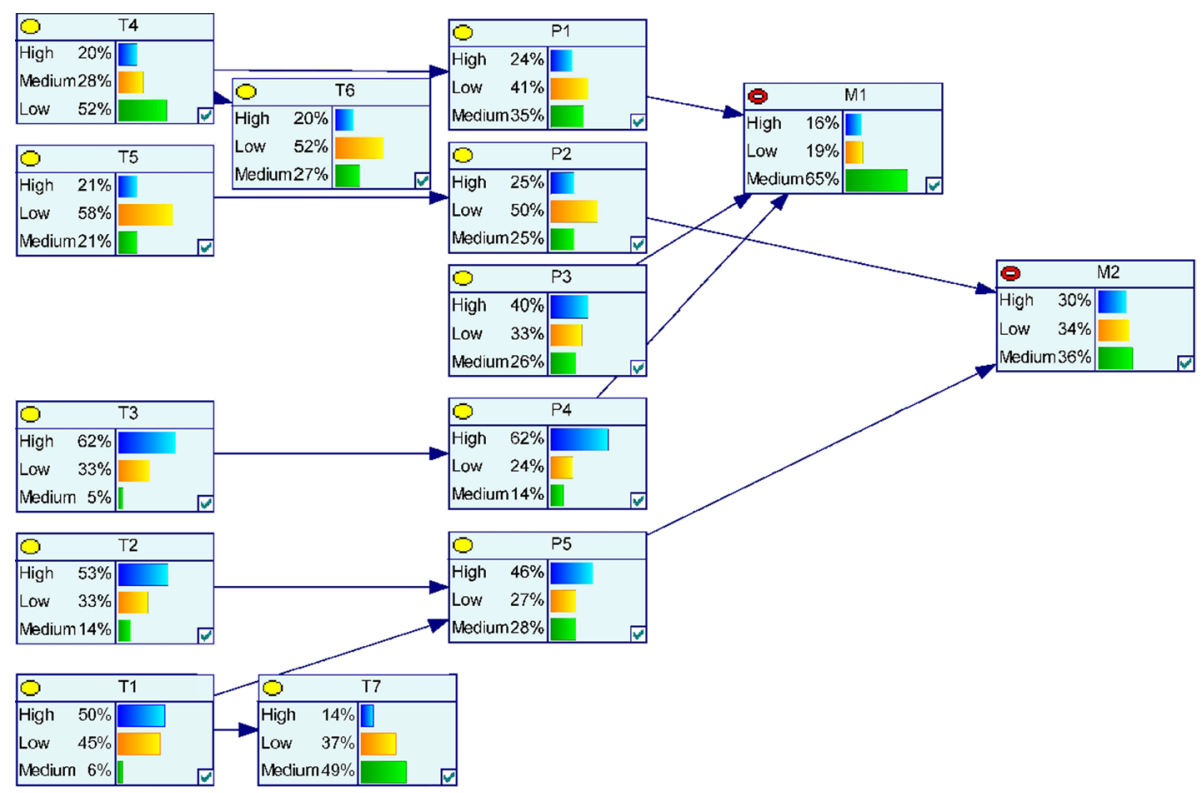

Fig. 4 The bayesian network for established technology roadmap (without future risk events) 


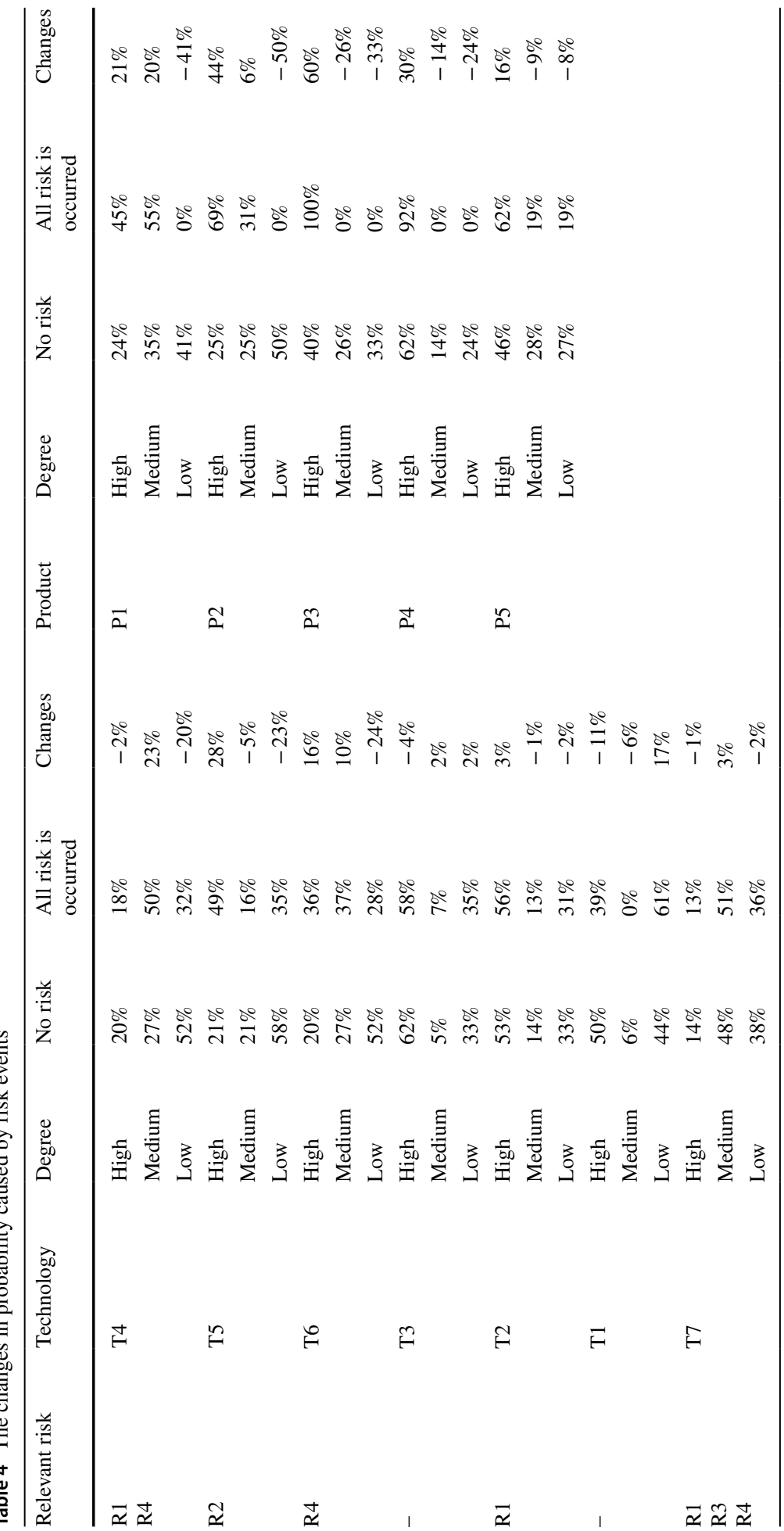




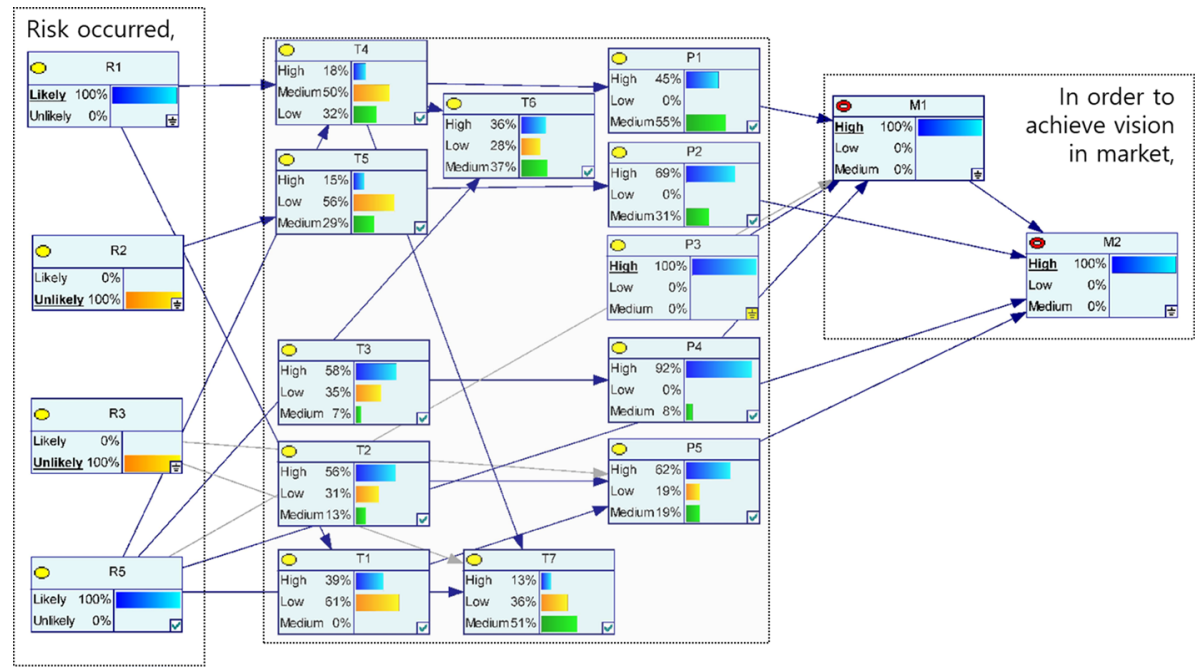

Fig. 5 The bayesian network for new technology roadmap with risk events

driver assistance), the probabilities of development decreased. All product nodes showed increased possibilities of development. In particular, the nodes for which the possibility decreased by risk event occurrence required alternative plans for responding to the event, which is derived at the next step.

\section{Risk-adaptive plans identified}

For nodes T1, T4, and T7, we found adaptive plans by co-occurrence of keywords between technology documents and risk documents. For instance, platooning was identified as an adaptive plan for climate change through text mining and analysis of the relationships between futuristic climate change data and technology, product, and market documents. Platooning is a promising technology that entails a driver of an initial car leading other vehicles clustered as one group (or sequence). The lead vehicle sets the time and distance intervals between vehicles, which enables maintaining a constant speed. On a highway, the slipstream effect of the lead vehicle in platooning reduces wind resistance for the subsequent vehicles, which reduces energy waste. Ultimately, this can reduce effects of climate change such as global warming and fine dust pollutants in Asia as well as reducing operation costs.

Cloud computing is necessary and fundamental for communication between objects such as automobiles. Self-driving cars in particular require cloud computing for processing every piece of data that each vehicle generates on the road and transferring these data to each other to allow for operation without drivers. For instance, through cloud computing, vehicles can share information with driver and control systems to control driving speed and directions automatically and in real time (Fig. 6).

The Internet of Things is another core technology that makes driverless vehicle operation possible; it provides intelligent service regardless of drivers' intention depending on information that objects transmit to each other, and self-driving cars need this technology to provide new value for new customers. In particular, it is critical for interacting not only with other vehicles but also with other objects. This risk event has brought about a new 


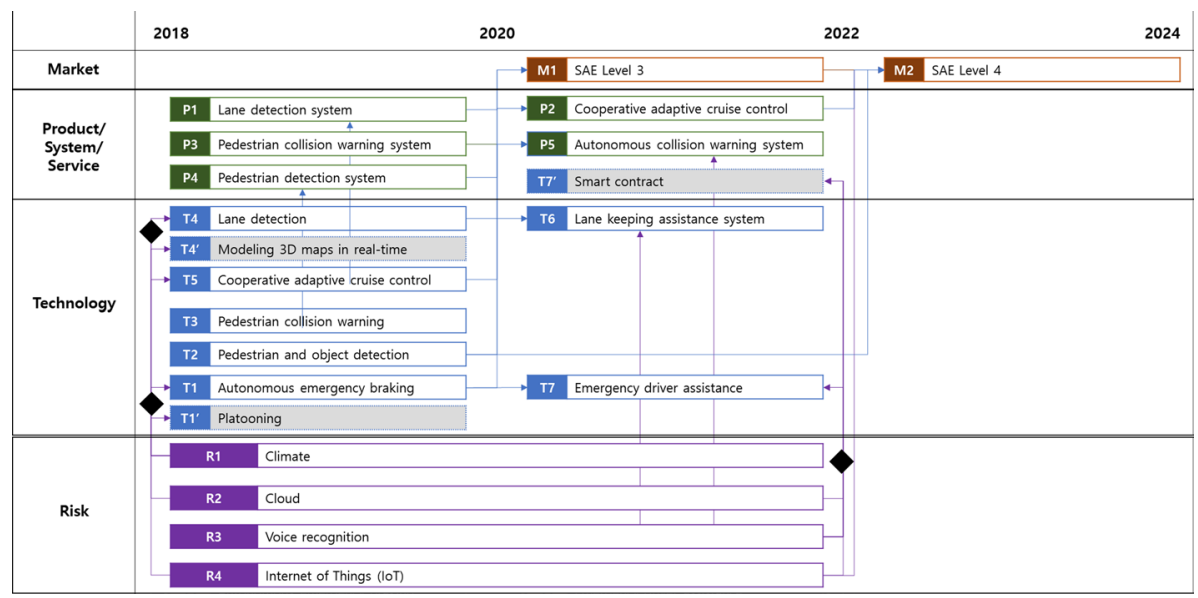

Fig. 6 The final technology roadmap

service, smart contracts, which allow for transactions to take place not through intermediaries such as cards or cash but by contacting specific parking points. Drivers do not need to leave their vehicles, which reduces time and effort to pay, ultimately improving efficiency on the road.

\section{Technology roadmapping}

To sum up, intermediary outcomes from each step are mapped on the existing technology roadmap but with some nodes that require decisions; the relationships between nodes for achieving visions are shown as market nodes on the map. The risk-adaptive roadmap is composed of four layers, technology, product (system, service), technology, and risk. The product layer is customized to the vehicle industry because self-driving cars require both tangible products and intangible services and thus any relevant system and service on the roadmap. In our study, we focused on four risks that have high probability of future occurrence and then found adaptive plans for these risk events. The adaptive plans were located as nodes on the technology roadmap with decision nodes for alternatives. The black diamonds on the map are decision nodes for when risk events occur; these nodes require decisions and then actions and for penetrating new markets, specifically for technology development. For an R1 event (climate change), platooning technology may be developed with T1 (autonomous emergency braking). R2 (cloud computing), R3 (voice recognition), and R4 (Internet of Things) encourage the generation of a new service, smart contracts (T7), which stemmed from T7 (emergency driver assistance).

\section{Validation and discussion}

The roadmapping results are validated by patent and market documents with experts who have worked in an automotive company for 3-5 years in two ways. They have relevant experience to find promising areas by analyzing patent documents and research papers, and roadmapping in practice with engineers in their affiliates. They evaluated 
that the overall direction of the suggested roadmap is well predicted, but they recommended an additional validation. The experts gave comments that each automobile company differently labels the title of technology and classifies the component technology implementing the self-driving vehicles according to their R\&D directions. Sometimes each firm sets the trajectory or sequences of technology development or targeting markets. Thus, we additionally validated by information retrieval using news articles and scholar databases (research papers, patents, and market reports in recent times). Moreover, we checked whether the risk and nodes on the roadmap occurred by investigating the trends of technology development after the risk-adaptive technology roadmap was developed. Thus, we validated again through information retrieval in recently published documents such as patents, research papers, and market reports after the domain experts evaluate at first.

At first, we searched news articles and market reports using the query including risks and nodes' contents that were mapped on the suggested roadmap to check whether the risk events really appeared within 2 years (from 2018 to 2020) and how the risks influence technology and market in automobile industry. While two years are too short to be published as a research paper or a patent document in general, news articles enable to identify the current issues immediately. Despite of a short haul, several risks identified from the proposed approach occurred and had an impact to self-driving cars-related market and technology.

The EU has already passed legislation to cut down CO2 emissions or heavy-duty vehicles (ICCT, 2019) and it brought about the necessity and urgency of truck platooning technology (Sivanandham \& Gajanand, 2020). In particular, the recent Covid-19 caused climate change and disrupt the structure of the market including the automobile industry. Helm (2020) discussed a set of questions related to the environmental impacts of coronavirus in a short and long-term with a global environmental policy. Many researchers and practitioners in automobile industry insist that automation and intelligence are essential to fight against the pandemic, especially autonomous driving makes ease the burden of covid-19 and climate change by supporting transportation which is necessary to supply medical resources and food to healthcare professionals and the public in infected areas (MIT Technology review, 2020). From the perspective of technology, even though the Covid-19 crisis delayed the development of advanced technologies such as autonomous driving and suspended driving testing (Hausler et al., 2020), vehicle platooning technologies have still developed and evolved continuously. When searching from USTPO database, 13 patents were retrieved (e.g. Patent US20200201674A1, Patent US10440668B1).

In the case of Internet of Things (IoTs), even though it has gotten attentions in recent times, the development of relevant technology such as blockchain technology and uncertain infectious diseases is stimulating interests on IoT and cloud computing. The number of businesses based on cloud computing and Internet of things has increased from 14 percent to about 25 percent in 2019 (Dahlqvist et al., 2019). With the development of blockchain technology, Internet of things, and cloud computing, infectious disease such as Covid-19 among risks accelerates non-contact service. Uncertain events are motivating new service generation such as smart contraction that runs autonomously when certain condition is fulfilled (Fraga-Lamas \& Fernandez-Carames, 2019). Like this way, several risks are already appeared in a short period with other risk factors. Based on these different risks, the change of markets and technology development are still in progress with a slow speed. Our proposed approach helps to find risk events and conduct adaptive plans. 


\section{Conclusion}

With this paper, we suggested a risk-adaptive technology roadmap for considering future uncertainty; the proposed map enables making new plans and actions when new risk or uncertainty occurs. The plans can be adapted to sudden changes in environment, and decision nodes in the roadmap help to select alternatives for achieving firm visions for the future. Our approach was based on a risk management framework of risk identification, evaluation, treatment, and feedback, and the roadmaps showed adaptive paths for maximizing outcomes following sudden risk events. The concept of risk-adaptive technology roadmap can be considered as an approach to make it possible to update and sustain the established technology roadmap despite of sudden risks through detecting potential risks and estimating impacts in advance from future databases including descriptions about the future status as longer as possible. The proposed approach assists to make strategies and plans for technology and product development in accordance with changing environments caused by future risks.

We used a Bayesian network and text mining including topic modeling to develop the risk-adaptive roadmap because Bayesian networks allow for considering relationships between nodes and make it possible to infer posterior probability. Other investigators have qualitatively illustrated interactions between nodes in Bayesian networks, but we estimated a conditional probability table based on documents related to technology, product, and market rather than using surveys or interviews. We estimated the degree of each node as a proxy using relationships between documents to consider real circumstances with uncertainty. This approach based on Bayesian network allowed for inferring future possibilities under uncertain environments.

In practice, the proposed roadmap can be applied to update and reuse established maps, which are difficult to update once they are set; for instance, if TRMs were developed in workshops, revising them could require another workshop or other large-scale work requiring additional investments of time, effort, and cost. Our proposed roadmap needs fewer additional resources than previous methods because it only incorporates new data into the new roadmap to update it. In particular, this study took advantage of databases that are opened to public such as patents and futuristic database. The methodology used for roadmapping in this paper, Bayesian network and topic modeling, has been widely used in recent times and techniques for these methods are already widespread and shared as open source. Thus, it enables to reconstruct our proposed method for ecosystem mapping with roadmap. The risk-adaptive roadmap will be helpful for agile marketing under rapidly changing circumstances; easily updated roadmaps reduce gaps between strategy planning and implementation by decreasing the time and effort needed to explore options.

However, our study has limitations that suggest further research in the future. For instance, it was our initial intention to consider external environment as much as possible by analyzing risks and future events, so we did not reflect internal factors such as costs and human resources and capabilities, but these factors could affect strategic directions given several adaptive plans. Second, the number of nodes might influence analysis; many nodes in a Bayesian network make it possible to analyze practical problems because of the increased complexity of the model itself. Third, one weakness of data-driven approaches is that incremental innovation is easier to achieve than breakthroughs through systematic analysis. Finally, the proposed risk-adaptive roadmap needs validation in practice, although we did illustrate its feasibility in the field of self-driving 
cars. In the near future, we will apply our approach to different industries to validate the risk-adaptive TRM's practical robustness.

Acknowledgement This work was supported by National Research Foundation of Korea (2019R1A2C1085388).

\section{References}

Abaei, M. M., Arzaghi, E., Abbassi, R., Garaniya, V., \& Penesis, I. (2017). Developing a novel risk-based methodology for multi-criteria decision making in marine renewable energy applications. Renewable Energy, 102, 341-348.

Adomavicius, G., Bockstedt, J. C., Gupta, A., \& Kauffman, R. J. (2008). Making sense of technology trends in the information technology landscape. Mis Quarterly, 32(4), 779.

Adomavicius, G., Bockstedt, J. C., Gupta, A., \& Kauffman, R. J. (2007). Technology roles and paths of influence in an ecosystem model of technology evolution. Information Technology and Management, $8(2), 185-202$

Albrechts, L. (2004). Strategic (spatial) planning reexamined. Environment and Planning B: Planning and design, 31(5), 743-758.

Amer, M., Daim, T. U., \& Jetter, A. (2016). Technology roadmap through fuzzy cognitive map-based scenarios: The case of wind energy sector of a developing country. Technology Analysis \& Strategic Management, 28(2), 131-155.

Ashrafi, M., Davoudpour, H., \& Khodakarami, V. (2017). A Bayesian network to ease knowledge acquisition of causal dependence in CREAM: Application of recursive noisy-OR gates. Quality and Reliability Engineering International, 33(3), 479-491.

Aven, T. (2012). Foundations of risk analysis. John Wiley \& Sons.

Butler, J. R. A., Wise, R. M., Skewes, T. D., Bohensky, E. L., Peterson, N., Suadnya, W., Yanuartati, Y., Handayani, T., Habibi, P., Puspadi, K., \& Bou, N. (2015). Integrating top-down and bottom-up adaptation planning to build adaptive capacity: A structured learning approach. Coastal Management, 43(4), 346-364.

Buurman, J., \& Babovic, V. (2016). Adaptation pathways and real options analysis: An approach to deep uncertainty in climate change adaptation policies. Policy and Society, 35(2), 137-150.

Carayannis, E. G., \& Campbell, D. F. (2009). "Mode 3'and'Quadruple Helix": Toward a 21st century fractal innovation ecosystem. International Journal of Technology Management, 46(3-4), 201-234.

Constantinou, A. C., Fenton, N. E., \& Neil, M. (2012). pi-football: A Bayesian network model for forecasting Association Football match outcomes. Knowledge-Based Systems, 36, 322-339.

Dahlqvist, F., Petal, M., Rajko, A., \& Shulman, J. (2019). Growing opportunities in the Internet of Things. Mckinsey. Retrieved from https://www.mckinsey.com/industries/private-equity-and-principal-inves tors/our-insights/growing-opportunities-in-the-internet-of-things.

de Melo, A. C., \& Sanchez, A. J. (2008). Software maintenance project delays prediction using Bayesian Networks. Expert Systems with Applications, 34(2), 908-919.

Firat, A. K., Woon, W. L., \& Madnick, S. (2008). Technological forecasting-A review. Composite Information Systems Laboratory (CISL), Massachusetts Institute of Technology.

Fraga-Lamas, P., \& Fernández-Caramés, T. M. (2019). A review on blockchain technologies for an advanced and cyber-resilient automotive industry. IEEE Access, 7, 17578-17598.

Garcia, M. L., \& Bray, O. H. (1997). Fundamentals of technology roadmapping (No. SAND-97-0665). Sandia National Labs., Albuquerque, NM (United States).

Gailis, R., Gunatilaka, A., Lopes, L., Skvortsov, A., \& Smith-Miles, K. (2014). Managing uncertainty in early estimation of epidemic behaviors using scenario trees. IIE Transactions, 46(8), 828-842.

Geum, Y., Lee, S., \& Park, Y. (2014). Combining technology roadmap and system dynamics simulation to support scenario-planning: A case of car-sharing service. Computers \& Industrial Engineering, 71, 37-49.

Geum, Y., Lee, H., Lee, Y., \& Park, Y. (2015). Development of data-driven technology roadmap considering dependency: An ARM-based technology roadmapping. Technological Forecasting and Social Change, 91, 264-279.

Haasnoot, M., Middelkoop, H., Offermans, A., Van Beek, E., \& Van Deursen, W. P. (2012). Exploring pathways for sustainable water management in river deltas in a changing environment. Climatic Change, 115(3-4), 795-819. 
Haasnoot, M., Kwakkel, J. H., Walker, W. E., \& ter Maat, J. (2013). Dynamic adaptive policy pathways: A method for crafting robust decisions for a deeply uncertain world. Global Environmental Change, 23(2), 485-498.

Hamarat, C., Kwakkel, J. H., \& Pruyt, E. (2013). Adaptive robust design under deep uncertainty. Technological Forecasting and Social Change, 80(3), 408-418.

Hansen, C., Daim, T., Ernst, H., \& Herstatt, C. (2016). The future of rail automation: A scenario-based technology roadmap for the rail automation market. Technological Forecasting and Social Change, $110,196-212$.

Hausler, S., Heineke, K., Hensley, R., Möller, T., Schwedhelm, D., \& Shen, P. (2020). The impact of COVID-19 on future mobility solutions. Mckinsey \& Company.

Helm, D. (2020). The environmental impact of the coronavirus. Environmental and Resource Economics, 76, 21-38.

Hu, Y., Zhang, X., Ngai, E. W. T., Cai, R., \& Liu, M. (2013). Software project risk analysis using Bayesian networks with causality constraints. Decision Support Systems, 56, 439-449.

Ilevbare, I. M., Probert, D., \& Phaal, R. (2014). Towards risk-aware roadmapping: Influencing factors and practical measures. Technovation, 34(8), 399-409.

International Council on Clean Transportation (2019). CO2 standards for heavy-duty vehicles in the European Union. Retrieved from https://theicct.org/publications/co2-stds-hdv-eu-20190416.

Islam, R., Khan, F., \& Venkatesan, R. (2017). Real time risk analysis of kick detection: Testing and validation. Reliability Engineering \& System Safety, 161, 25-37.

Jackson, D. J. (2011). What is an innvovation ecosystem. National Science Foundation, 1, 1-13.

Jin, G., Jeong, Y., \& Yoon, B. (2015). Technology-driven roadmaps for identifying new product/market opportunities: Use of text mining and quality function deployment. Advanced Engineering Informatics, 29(1), 126-138.

Kabir, M. J., Alauddin, M., \& Crimp, S. (2017). Farm-level adaptation to climate change in Western Bangladesh: An analysis of adaptation dynamics, profitability and risks. Land Use Policy, 64, $212-224$.

Kaplan, S., \& Garrick, B. J. (1981). On the quantitative definition of risk. Risk analysis, 1(1), 11-27.

Khakzad, N. (2015). Application of dynamic Bayesian network to risk analysis of domino effects in chemical infrastructures. Reliability Engineering \& System Safety, 138, 263-272.

Khakzad, N., Khan, F., \& Amyotte, P. (2013). Dynamic safety analysis of process systems by mapping bow-tie into Bayesian network. Process Safety and Environmental Protection, 91(1-2), 46-53.

Kim, J., Han, M., Lee, Y., \& Park, Y. (2016). Futuristic data-driven scenario building: Incorporating text mining and fuzzy association rule mining into fuzzy cognitive map. Expert Systems with Applications, 57, 311-323.

Kostoff, R. N., \& Schaller, R. R. (2001). Science and technology roadmaps. IEEE Transactions on Engineering Management, 48(2), 132-143.

Krüger, C., \& Lakes, T. (2015). Bayesian belief networks as a versatile method for assessing uncertainty in land-change modeling. International Journal of Geographical Information Science, 29(1), 111-131.

Kwakkel, J. H., Auping, W. L., \& Pruyt, E. (2013). Dynamic scenario discovery under deep uncertainty: The future of copper. Technological Forecasting and Social Change, 80(4), 789-800.

Lahoti, G., Porter, A. L., Zhang, C., Youtie, J., \& Wang, B. (2018). Tech mining to validate and refine a technology roadmap. World Patent Information, 55, 1-18.

Lawrence, J., \& Haasnoot, M. (2017). What it took to catalyse uptake of dynamic adaptive pathways planning to address climate change uncertainty. Environmental Science \& Policy, 68, 47-57.

Lee, C., Song, B., \& Park, Y. (2015). An instrument for scenario-based technology roadmapping: How to assess the impacts of future changes on organisational plans. Technological Forecasting and Social Change, 90, 285-301.

Lee, H., \& Geum, Y. (2017). Development of the scenario-based technology roadmap considering layer heterogeneity: An approach using CIA and AHP. Technological Forecasting and Social Change, 117, 12-24.

Lee, M., \& Song, M. (2020). Incorporating citation impact into analysis of research trends. Scientometrics, 124, 1191-1224.

Lee, S., \& Park, Y. (2005). Customization of technology roadmaps according to roadmapping purposes: Overall process and detailed modules. Technological Forecasting and Social Change, 72(5), 567-583.

Lempert, R., Nakicenovic, N., Sarewitz, D., \& Schlesinger, M. (2004). Characterizing climate-change uncertainties for decision-makers. Climatic Change, 65(1), 1-9.

Li, M., \& Porter, A. (2018). Facilitating the discovery of relevant studies on risk analysis for threedimensional printing based on an integrated framework. Scientometrics, 114, 277-300. 
Li, Y. R. (2009). The technological roadmap of Cisco's business ecosystem. Technovation, 29(5), $379-386$

Maier, H. R., Guillaume, J. H., van Delden, H., Riddell, G. A., Haasnoot, M., \& Kwakkel, J. H. (2016). An uncertain future, deep uncertainty, scenarios, robustness and adaptation: How do they fit together? Environmental Modelling \& Software, 81, 154-164.

Marrone, M. (2020). Application of entity linking to identify research fronts and trends. Scientometrics, 122, 357-379.

MIT Technology Review (2020). How coronavirus is accelerating a future with autonomous vehicles. Retrieved from https://www.technologyreview.com/2020/05/18/1001760/how-coronavirus-is-accel erating-autonomous-vehicles/.

Offermans, A., \& Cörvers, R. (2012). Learning from the past; changing perspectives on river management in the Netherlands. Environmental Science \& Policy, 15(1), 13-22.

Park, H., Phaal, R., Ho, J., \& O’Sullivan, E. (2020). Twenty years of technology and strategic roadmapping research: A school of thought perspective. Technological Forecasting and Social Change, 154, 119965.

Phaal, R., Farrukh, C. J., \& Probert, D. R. (2004). Technology roadmapping-A planning framework for evolution and revolution. Technological Forecasting and Social Change, 71(1-2), 5-26.

Phaal, R., Farrukh, C. J., \& Probert, D. R. (2005). Developing a technology roadmapping system. Unifying Discipline for Melting the Boundaries Technology Management, (pp. 99-111). IEEE.

Phaal, R., Farrukh, C. J., \& Probert, D. R. (2007). Strategic roadmapping: A workshop-based approach for identifying and exploring strategic issues and opportunities. Engineering Management Journal, 19(1), 3-12.

Phaal, R., \& Muller, G. (2009). An architectural framework for roadmapping: Towards visual strategy. Technological Forecasting and Social Change, 76(1), 39-49.

Purba, J. H., Tjahyani, D. S., Ekariansyah, A. S., \& Tjahjono, H. (2015). Fuzzy probability based fault tree analysis to propagate and quantify epistemic uncertainty. Annals of Nuclear Energy, 85, 1189-1199.

Ranaei, S., Suominen, A., \& Porter, A. (2020). Evaluating technological emergence using text analytics: Two case technologies and three approaches. Scientometrics, 122, 215-247.

Ranger, N., Millner, A., Dietz, S., Fankhauser, S., Lopez, A., \& Ruta, G. (2010). Adaptation in the UK: A decision-making process. Environment Agency, 9, 1-62.

Rinne, M. (2004). Technology roadmaps: Infrastructure for innovation. Technological Forecasting and Social Change, 71(1-2), 67-80.

Rosenzweig, C., \& Solecki, W. (2014). Hurricane Sandy and adaptation pathways in New York: Lessons from a first-responder city. Global Environmental Change, 28, 395-408.

Saritas, O., \& Aylen, J. (2010). Using scenarios for roadmapping: The case of clean production. Technological Forecasting and Social Change, 77(7), 1061-1075.

Siebelink, R., Halman, J. I., \& Hofman, E. (2016). Scenario-Driven roadmapping to cope with uncertainty: Its application in the construction industry. Technological Forecasting and Social Change, 110, 226-238.

Sivanandham, S., \& Gajanand, M. S. (2020). Platooning for sustainable freight transportation: an adoptable practice in the near future? Transport Reviews. https://doi.org/10.1080/01441647.2020.1747568

Strauss, J. D., \& Radnor, M. (2004). Roadmapping for dynamic and uncertain environments. Research-Technology Management, 47(2), 51-58.

Van der Brugge, R., Rotmans, J., \& Loorbach, D. (2005). The transition in Dutch water management. Regional Environmental Change, 5(4), 164-176.

Walker, W., Haasnoot, M., \& Kwakkel, J. (2013). Adapt or perish: A review of planning approaches for adaptation under deep uncertainty. Sustainability, 5(3), 955-979.

Wang, G., Xu, T., Tang, T., Yuan, T., \& Wang, H. (2017). A Bayesian network model for prediction of weather-related failures in railway turnout systems. Expert Systems with Applications, 69, 247-256.

Xu, G., Wu, Y., Minshall, T., \& Zhou, Y. (2018). Exploring innovation ecosystems across science, technology, and business: A case of 3D printing in China. Technological Forecasting and Social Change, 136, 208-221.

Yet, B., Constantinou, A., Fenton, N., Neil, M., Luedeling, E., \& Shepherd, K. (2016). A Bayesian network framework for project cost, benefit and risk analysis with an agricultural development case study. Expert Systems with Applications, 60, 141-155.

Yohe, G., \& Toth, F. L. (2000). Adaptation and the guardrail approach to tolerable climate change. Climatic Change, 45(1), 103-128.

Zhang, X., Fan, J. L., \& Wei, Y. M. (2013). Technology roadmap study on carbon capture, utilization and storage in China. Energy Policy, 59, 536-550. 